\title{
Non-Associative Geometry of Quantum Tori
}

\author{
Francesco D'ANDREA ${ }^{\dagger \ddagger}$ and Davide FRANCO \\ † Dipartimento di Matematica e Applicazioni, Università di Napoli "Federico II", \\ Complesso MSA, Via Cintia, 80126 Napoli, Italy \\ $\ddagger$ I.N.F.N. Sezione di Napoli, Complesso MSA, Via Cintia, 80126 Napoli, Italy \\ E-mail: francesco.dandrea@unina.it,dfranco@unina.it
}

Received October 02, 2015, in final form February 04, 2016; Published online February 07, 2016

http://dx.doi.org/10.3842/SIGMA.2016.015

\begin{abstract}
We describe how to obtain the imprimitivity bimodules of the noncommutative torus from a "principal bundle" construction, where the total space is a quasi-associative deformation of a 3-dimensional Heisenberg manifold.
\end{abstract}

Key words: noncommutative torus; quasi-Hopf algebras; cochain quantization

2010 Mathematics Subject Classification: 58B34; 46L87; 53D55

\section{Introduction}

In differential geometry a standard way to construct vector bundles is from a principal bundle and a representation of the structure group. This construction works in noncommutative geometry too $[6,13,15]$, with spaces replaced by algebras, vector bundles replaced by finitely generated projective modules, structure groups replaced by Hopf algebras or compact quantum groups, and principal bundles replaced by algebra extensions with suitable additional properties (see, e.g., $[3,14]$ ). When the structure group is $U(1)$, it is possible to reconstruct the total space of the "bundle" (more precisely, a strongly graded $C^{*}$-algebra) from the base space and a noncommutative "line bundle" (a self-Morita equivalence bimodule), cf. [2] (see also [1, 7]).

A case study is provided by the $C^{*}$-algebra of the noncommutative torus $A_{\theta}, \theta \in \mathbb{R} \backslash \mathbb{Q}[5,20]$. The group $\mathrm{SL}_{2}(\mathbb{Z})$ acts on $\mathbb{R} \backslash \mathbb{Q}$ by fractional linear transformations,

$$
g \theta:=\frac{a \theta+b}{c \theta+d}, \quad \forall \theta \in \mathbb{R} \backslash \mathbb{Q} \quad \text { and } \quad g=\left(\begin{array}{ll}
a & b \\
c & d
\end{array}\right) \in \mathrm{SL}_{2}(\mathbb{Z}),
$$

and for every $g$ as above there is a full right Hilbert $A_{\theta}$-module $E_{g}(\theta)$ which is a Morita equivalence bimodule between $A_{\theta}$ and the algebra $\operatorname{End}_{A_{\theta}}\left(E_{g}(\theta)\right) \simeq A_{g \theta}[5,21]$. The equivalence class of the right module $E_{g}(\theta)$ only depends on the 'degree' and 'rank' of the module, defined by $\operatorname{deg}\left(E_{g}(\theta)\right)=c$ and $\operatorname{rank}\left(E_{g}(\theta)\right)=c \theta+d$ (see, e.g., [19]). Every other finitely generated projective right $A_{\theta}$-module is a direct sum of such modules.

In this paper we show how one can formally derive some of these bimodules - those with rank 1 for $\theta \rightarrow 0$ (hence, line bundles) - from some kind of $U(1)$-bundle, but at the price of working with quasi-associative algebras, i.e., monoids in the category of representations of a quasi-Hopf algebra. Several well-known properties of these bimodules can be shown to be a consequence of the explicit form of the coassociator (cf. Lemma 3.1), including the associativity property of the pairing of bimodules of Polishchuk and Schwarz [19].

The plan of the paper is the following. In Section 2 we recall some basic definitions about twist deformations of quasi-Hopf algebras and algebra modules. In Section 3 we will introduce the twist we are interested in, based on the universal enveloping algebra of the 3-dimensional Heisenberg Lie algebra $\mathfrak{h}_{3}(\mathbb{R})$; we will also prove some generalized associativity property of 


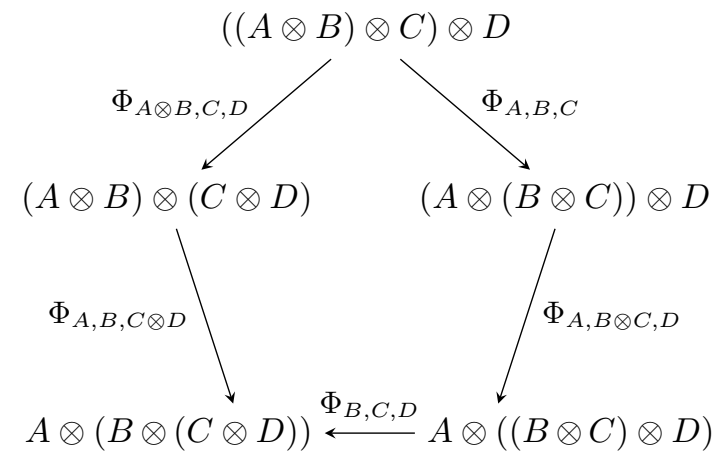

(a) Pentagon diagram

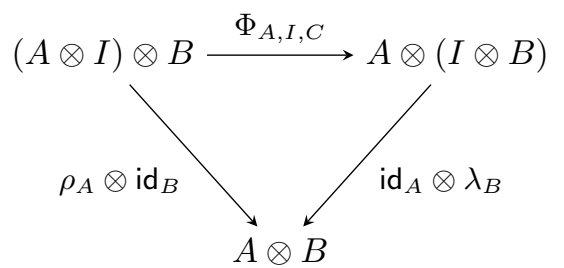

(b) Triangle diagram

Figure 1

twisted module algebras, namely Proposition 3.2. In Section 4 we will give a concrete realization of $\mathfrak{h}_{3}(\mathbb{R})$ in terms of differential operators on the total space of a principal $U(1)$-bundle $M_{3} \rightarrow \mathbb{T}^{2}$ on the 2-torus, and apply to it the deformation recipe of Section 2 with the twist introduced in Section 3: the total space becomes a quasi-associative $\mathbb{Z}$-graded algebra, which in degree 0 is the algebra of smooth functions on the noncommutative torus; in degree $n \neq 0$ we get bimodules that we compare with Connes-Rieffel imprimitivity bimodules. In Section 5 we give a slightly different version of the construction, more natural if one is interested in complex structures: we show that, in this case, the twisted algebra of functions on the Heisenberg manifold has an associative commutative subalgebra given by ordinary theta functions. In Section 6 we study vector bundles of any rank.

Notations. By a algebra we shall always mean a unital algebra (not necessarily associative nor commutative) over a commutative unital ring $R$; the algebraic tensor product over $R$ will be denoted by $\otimes_{R}$, or simply $\otimes$ if there is no risk of confusion; by a Hopf algebra over $\mathbb{C}[[\nu]]$ we shall always mean a topological Hopf algebra, completed in the $h$-adic topology, and by $\otimes_{\mathbb{C}[[\nu]]}$ the completed tensor product [4, Section 4.1A].

\section{Mathematical preliminaries}

In this section, we recall some definitions and properties of quasi-Hopf algebras and Drinfeld twists, from $[4,10,11,16]$.

\subsection{Monoidal categories}

A monoidal category is a category $\mathbf{C}$ equipped with a functor $\otimes: \mathbf{C} \times \mathbf{C} \rightarrow \mathbf{C}$ which, modulo natural isomorphisms, is associative and unital [4, Section 5.1]. More precisely, there is an object $I$ and three natural isomorphisms $-\Phi$ between the functors $\left(_{-} \otimes_{-}\right) \otimes_{-}$and $\left.\otimes_{-} \otimes_{-} \otimes_{-}\right)$, $\lambda$ between $I \otimes_{-}$and the identity, $\rho$ between $\_$- $\otimes$ and the identity - such that the diagrams 1a and $1 \mathrm{~b}$ commute for all objects $A, B, C, D$. Examples are the category of modules over a field (vector spaces), over a group or a Hopf algebra (representations), over a topological Hopf algebra (with completed tensor product).

A monoid [16, Section VII.3] in a monoidal category is an object $A$ together with two arrows $m: A \otimes A \rightarrow A$ and $\eta: I \rightarrow A$, the "multiplication" and "unit", satisfying the usual axioms of a unital associative algebra modulo natural transformations, namely the diagrams $2 \mathrm{a}$ and $2 \mathrm{~b}$ must commute. Monoids in the category of vector spaces are associative algebras, in the category of representations of a Hopf algebra $H$ are $H$-module algebras. 


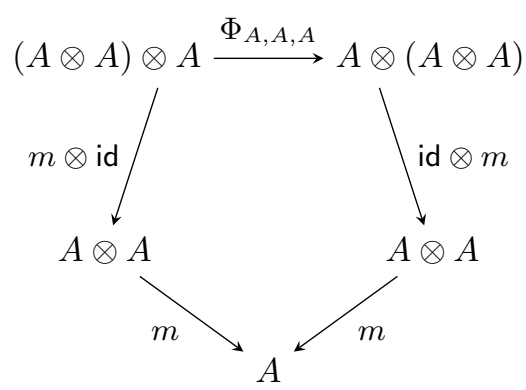

(a) Associativity

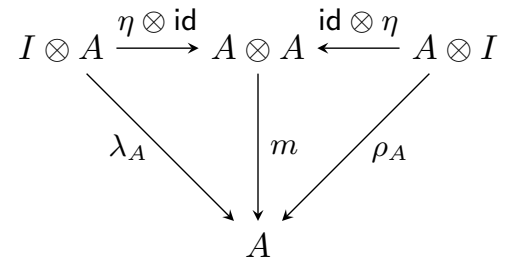

(b) Unitality

Figure 2

\subsection{Quasi-Hopf algebras}

Let $H$ be an algebra and $\Delta: H \rightarrow H \otimes H$ a linear map. For all $n \geq m$ and all $1 \leq i_{1}<i_{2}<\cdots<$ $i_{m} \leq n$, we will denote by $h \mapsto h_{i_{1} \ldots i_{m}}$ the linear map $H^{\otimes m} \rightarrow H^{\otimes n}$ defined on homogeneous tensors $h=a_{1} \otimes a_{2} \otimes \cdots \otimes a_{m}$ as follows: we put $a_{k}$ in the leg $i_{k}$ of the tensor product for all $k=1, \ldots, m$, and fill the additional $n-m$ legs with 1 . The subscript $\left(i_{k} i_{k+1}\right)$ in parenthesis means that we apply $\Delta$ to $a_{k}$ and put the first leg of $\Delta\left(a_{k}\right)$ in position $i_{k}$ and the second in position $i_{k+1}$. So, for example, if $h=a \otimes b, h_{13}=a \otimes 1 \otimes b, h_{(12) 3}=\Delta(a) \otimes b$, etc.

A quasi-bialgebra (over a commutative ring $R$ ) is an associative algebra $H$ together with two homomorphisms $\Delta: H \rightarrow H \otimes H$ and $\epsilon: H \rightarrow R$ (the "coproduct" and "counit") and an invertible element $\Phi \in H \otimes H \otimes H$ (the "coassociator") satisfying the following conditions (here we follow the notations of $[10,11])$ :

$$
\begin{aligned}
& \Phi_{12(34)} \Phi_{(12) 34}=\Phi_{234} \Phi_{1(23) 4} \Phi_{123}, \\
& (\text { id } \otimes \epsilon \otimes \text { id })(\Phi)=1,
\end{aligned}
$$

and

$$
\begin{aligned}
& (\text { id } \otimes \Delta) \Delta(h)=\Phi(\Delta \otimes \text { id }) \Delta(h) \Phi^{-1}, \\
& (\text { id } \otimes \epsilon) \Delta(h)=h=(\epsilon \otimes \text { id }) \Delta(h),
\end{aligned}
$$

for all $h \in H$. A quasi-Hopf algebra is a quasi-bialgebra satisfying an additional condition which for $\Phi=1$ reduces to the existence and bijectivity of an antipode, see, e.g., [4, Definition 16.1.1].

Any Hopf algebra is a quasi-Hopf algebra with trivial coassociator, $\Phi=1$. In fact, in the Hopf case any $\Phi$ which is invariant (i.e., commutes with the image of the iterated coproduct) does the job. If $\Phi$ is invariant, (2.3) reduces to the coassociativity condition (id $\otimes \Delta) \Delta=(\Delta \otimes$ id) $\Delta$ and we get the usual definition of bialgebra/Hopf algebra.

If $H$ is a commutative Hopf algebra, (2.1) can be interpreted as a 3-cocycle condition in the Hopf algebra cohomology of $H$ [17, Section 2.3]. In general, if $A, B, C$ are three $H$-modules, we may think of $\Phi$ as a module map $(A \otimes B) \otimes C \rightarrow A \otimes(B \otimes C)$, with the natural isomorphism of vector spaces understood. Condition (2.1) becomes the associativity condition 1a for the category of $H$-modules, which is then monoidal with unit object $I=R$ given by the ground ring (with module structure being given by the counit, so that (2.2) implies the commutativity of the diagram $1 \mathrm{~b}$, and module structure on a tensor product defined by the coproduct).

A monoid $A$ in this category is a $H$-module algebra, that is an algebra $A$ with multiplication satisfying the quasi-associativity condition (cf. diagram 2a):

$$
m(m \otimes \mathrm{id})=m(\mathrm{id} \otimes m) \Phi,
$$

Commutativity of the diagram $2 \mathrm{~b}$ means that $A$ is unital, with $1_{A}:=\eta\left(1_{R}\right)$. The condition that $m$ and $\eta$ are morphisms in the category, i.e., $H$-module maps, gives $h .1_{A}=\epsilon(h) 1_{A}$ and

$$
h \circ m=m \circ \Delta(h),
$$


for all $h \in H$. Equation (2.4) becomes the usual associativity condition if the action of $\Phi$ on $A \otimes A \otimes A$ is trivial (a sufficient condition of course is that $\Phi=1$ ).

The above definitions remain valid if the ground ring is $R=\mathbb{C}[[\nu]]$, with $\otimes_{R}$ the completed tensor product.

The advantage of quasi-bialgebras (resp. quasi-Hopf algebras) over ordinary bialgebras (resp. Hopf algebras) is that there is a "gauge action" of the group of invertible elements in $H \otimes H$ that doesn't change the category of modules.

Let $H$ be a quasi-bialgebra (resp. quasi-Hopf algebra) and $F \in H \otimes H$ an invertible element satisfying (id $\otimes \epsilon)(F)=(\epsilon \otimes$ id $)(F)=1$. One can define a new quasi-bialgebra (resp. quasi-Hopf algebra) $H_{F}$ given by $H$ as an algebra and with the same counit, but with a new coproduct $\Delta_{F}$ and coassociator $\Phi_{F}$ defined by

$$
\begin{aligned}
& \Delta_{F}(h):=F \Delta(h) F^{-1}, \quad \forall h \in H, \\
& \Phi_{F}:=F_{23} F_{1(23)} \Phi\left(F^{-1}\right)_{(12) 3}\left(F^{-1}\right)_{12} .
\end{aligned}
$$

It turns out that the categories of $H$-modules and $H_{F}$-modules are equivalent as monoidal categories. In particular, if $(A, m)$ is a $H$-module algebra, there is a $H_{F}$-module algebra $\left(A, m_{F}\right)$ given by $A$ as a vector space, with the same unit element, and with multiplication:

$$
m_{F}:=m \circ F^{-1} \text {. }
$$

We will refer to $H_{F}$ as a twist deformation of $H$, and to $F$ as a twisting element based on $H$.

\subsection{Quantum universal enveloping algebras}

By a deformation of a quasi-bialgebra over a field $\mathbb{k}$ we mean a topological quasi-bialgebra $H_{\nu}$ over $\mathbb{k}[[\nu]]$ such that $H_{\nu} / \nu H_{\nu} \simeq H$ as quasi-bialgebras and $H_{\nu} \simeq H[[\nu]]$ as $\mathbb{k}[[\nu]]$-modules. If $H$ is a quasi-Hopf algebra, any deformation as a quasi-bialgebra is in fact a quasi-Hopf algebra [4, Section $16.1 \mathrm{C}]$.

If $H=\mathcal{U}(\mathfrak{g})$ is the universal enveloping algebra of a Lie algebra $\mathfrak{g}$, with standard Hopf algebra structure and trivial coassociator, a deformation $H_{\nu}$ with coassociator $\Phi_{\nu} \equiv 1 \bmod \nu^{2}$ will be called a quantum universal enveloping algebra (or QUEA). Any twist of $\mathcal{U}(\mathfrak{g})$ by a twisting element $F_{\nu}$ based on $\mathcal{U}(\mathfrak{g})[[\nu]]$ (and satisfying $F_{\nu} \equiv 1 \bmod \nu$ ) is a QUEA, and roughly speaking every QUEA arises in this way (see Theorem 16.1.11 of [4] for the precise statement).

\section{A twist based on the Heisenberg Lie algebra}

The example which is of interest to us is based on the Lie algebra $\mathfrak{h}_{3}(\mathbb{R})$ of the 3-dimensional Heisenberg group, or more precisely on the Hopf algebra $\mathcal{U}\left(\mathfrak{h}_{3}(\mathbb{R})\right)$.

The Lie algebra $\mathfrak{h}_{3}(\mathbb{R})$ has a basis of three elements $\mathfrak{p}, \mathfrak{q}, \mathfrak{t}$, with $\mathfrak{t}$ central and

$$
[\mathfrak{p}, \mathfrak{q}]=\mathfrak{t} .
$$

Let us call parameter space $\Theta \subset \mathbb{C}[[\nu]]$ the ideal:

$$
\Theta:=\nu \mathbb{C}[[\nu]]
$$

Note that for all $\left\{a_{n}\right\}_{n \geq 0}$ belonging to a complex vector space $A$ and for all $\theta \in \Theta$, the expression $\sum_{n \geq 0} a_{n} \theta^{n}$ is a well defined element of $A[[\nu]]$ (for each $N \geq 0$, the coefficient of $\nu^{N}$ is a finite linear combination of elements of $A$ ). 
There is an action $\alpha$ of $\mathbb{Z}$ by automorphisms on the vector space $\Theta$ given by

$$
\alpha_{k}(\theta)=\frac{\theta}{1+k \theta}=\theta-k \theta^{2}+\cdots, \quad k \in \mathbb{Z}, \quad \theta \in \Theta
$$

Clearly $\alpha$ maps $\Theta$ into itself, and $\alpha_{j} \alpha_{k}=\alpha_{j+k} \forall j, k \in \mathbb{Z}$.

For all $\theta \in \Theta$, a twisting element based on $\mathcal{U}\left(\mathfrak{h}_{3}(\mathbb{R})\right)[[\nu]]$ is

$$
F_{\theta}=\exp \{\theta \mathfrak{p} \otimes \mathfrak{q}\} .
$$

Let $A$ be any $\mathcal{U}\left(\mathfrak{h}_{3}(\mathbb{R})\right)$-module algebra (associative, since the coassociator is trivial), and for all $n \in \mathbb{Z}$ define

$$
A_{n}:=\{a \in A: \mathfrak{t} . a=n a\} .
$$

Since $\mathfrak{t}$ is central, each $A_{n}$ is a $\mathcal{U}\left(\mathfrak{h}_{3}(\mathbb{R})\right.$ )-module itself, and $A_{\bullet}:=\bigoplus_{n \in \mathbb{Z}} A_{n}$ is a graded (associative) module subalgebra of $A$.

We let $a *_{\theta} b:=m \circ F_{\theta}^{-1}(a \otimes b)$ be the product on $A[[\nu]]$ defined in (2.5), and denote by $A^{\theta}=\left(A[[\nu]], *_{\theta}\right)$ the deformed algebra. Since $\mathfrak{t}$ is central, each set $A_{n}[[\nu]]$ is stable under the action of $\mathfrak{p}, \mathfrak{q}$. So $A_{\bullet}^{\theta}=\left(A_{\bullet}[[\nu]], *_{\theta}\right)$ is a $\mathbb{Z}$-graded subalgebra of $A^{\theta}$, and $A_{0}^{\theta}=\left(A_{0}[[\nu]], *_{\theta}\right)$ is a yet smaller subalgebra.

Lemma 3.1. For all $\theta, \theta^{\prime} \in \Theta$ :

$$
(\Delta \otimes \mathrm{id})\left(F_{\theta}^{-1}\right)\left(F_{\theta^{\prime}}^{-1} \otimes 1\right)=(\mathrm{id} \otimes \Delta)\left(F_{\theta^{\prime}}^{-1}\right)\left(1 \otimes F_{\theta}^{-1}\right) \Phi_{\theta, \theta^{\prime}}
$$

where

$$
\Phi_{\theta, \theta^{\prime}}:=\exp \left\{-\mathfrak{p} \otimes\left(\theta-\theta^{\prime}-\theta \theta^{\prime} \mathfrak{t}\right) \otimes \mathfrak{q}\right\} .
$$

Proof. From (3.4), recalling that $\mathfrak{p}, \mathfrak{q}$ are primitive elements

$$
\begin{aligned}
\Phi_{\theta, \theta^{\prime}} & =\left(1 \otimes F_{\theta}\right)(\mathrm{id} \otimes \Delta)\left(F_{\theta^{\prime}}\right)(\Delta \otimes \mathrm{id})\left(F_{\theta}^{-1}\right)\left(F_{\theta^{\prime}}^{-1} \otimes 1\right) \\
& =e^{\theta 1 \otimes \mathfrak{p} \otimes \mathfrak{q}} e^{\theta^{\prime}(\mathfrak{p} \otimes 1 \otimes \mathfrak{q}+\mathfrak{p} \otimes \mathfrak{q} \otimes 1)} e^{-\theta(\mathfrak{p} \otimes 1 \otimes \mathfrak{q}+1 \otimes \mathfrak{p} \otimes \mathfrak{q})} e^{-\theta^{\prime} \mathfrak{p} \otimes \mathfrak{q} \otimes 1} .
\end{aligned}
$$

Next, we use Baker-Campbell-Hausdorff formula, which for two elements $X, Y$ of an associative algebra with central commutator $[X, Y]$ reduces to $e^{\nu X} e^{\nu Y}=e^{\nu^{2}[X, Y]} e^{\nu Y} e^{\nu X}$. We get

$$
e^{-\theta(\mathfrak{p} \otimes 1 \otimes \mathfrak{q}+1 \otimes \mathfrak{p} \otimes \mathfrak{q})} e^{-\theta^{\prime} \mathfrak{p} \otimes \mathfrak{q} \otimes 1}=e^{\theta \theta^{\prime} \mathfrak{p} \otimes \mathfrak{t} \otimes \mathfrak{q}} e^{-\theta^{\prime} \mathfrak{p} \otimes \mathfrak{q} \otimes 1} e^{-\theta(\mathfrak{p} \otimes 1 \otimes \mathfrak{q}+1 \otimes \mathfrak{p} \otimes \mathfrak{q})} .
$$

Using this in (3.6) we get

$$
\begin{aligned}
\Phi_{\theta, \theta^{\prime}} & =e^{\theta \theta^{\prime} \mathfrak{p} \otimes \mathfrak{t} \otimes \mathfrak{q}} e^{\theta 1 \otimes \mathfrak{p} \otimes \mathfrak{q}}\left(e^{\theta^{\prime}(\mathfrak{p} \otimes 1 \otimes \mathfrak{q}+\mathfrak{p} \otimes \mathfrak{q} \otimes 1)} e^{-\theta^{\prime} \mathfrak{p} \otimes \mathfrak{q} \otimes 1}\right) e^{-\theta(\mathfrak{p} \otimes 1 \otimes \mathfrak{q}+1 \otimes \mathfrak{p} \otimes \mathfrak{q})} \\
& =e^{\theta \theta^{\prime} \mathfrak{p} \otimes \mathfrak{t} \otimes \mathfrak{q}} e^{\theta 1 \otimes \mathfrak{p} \otimes \mathfrak{q}} e^{\theta^{\prime} \mathfrak{p} \otimes 1 \otimes \mathfrak{q}} e^{-\theta(\mathfrak{p} \otimes 1 \otimes \mathfrak{q}+1 \otimes \mathfrak{p} \otimes \mathfrak{q})} .
\end{aligned}
$$

Now all the exponents mutually commute, and after some simplification we arrive at (3.5).

In particular, we can observe that the coassociator:

$$
\Phi_{\theta, \theta}=e^{\theta^{2} \mathfrak{p} \otimes \mathfrak{t} \otimes \mathfrak{q}}
$$

of the QUE algebra is not trivial nor invariant, so that we are dealing with a genuine quasi-Hopf deformation of $\mathcal{U}\left(\mathfrak{h}_{3}(\mathbb{R})\right)$. Many properties of $A^{\theta}$ can be deduced from Lemma 3.1. 
Proposition 3.2. If $\theta^{\prime}=\alpha_{n}(\theta)$, with $\alpha$ as in (3.2), one has the generalized associativity law:

$$
\left(a *_{\theta^{\prime}} b\right) *_{\theta} c=a *_{\theta^{\prime}}\left(b *_{\theta} c\right) \quad \forall a, c \in A[[\nu]], \quad b \in A_{n}[[\nu]] .
$$

Proof. If $\theta^{\prime}=\alpha_{n}(\theta)$, from the definition of the star product and the observation that $\mathfrak{t} . b=n b$, we deduce that $\Phi_{\theta, \theta^{\prime}}$ is the identity on $a \otimes b \otimes c$ and then (3.8) holds.

\section{Corollary 3.3.}

1) $A_{0}^{\theta}$ is an associative unital subalgebra of $A^{\theta}$.

2) $E_{n}:=A_{n}[[\nu]]$ is an $A_{0}^{\alpha_{n}(\theta)}-A_{0}^{\theta}$-bimodule (with left module structure given by $*_{\alpha_{n}(\theta)}$ and right module structure given by $*_{\theta}$ ).

3) $*_{\theta}: E_{j} \otimes E_{k} \rightarrow E_{j+k}$ descends to a map $E_{j} \otimes A_{0}^{\theta} E_{k} \rightarrow E_{j+k}$ (where the left and right module structure are given by the $*_{\theta}$ multiplication).

4) For all $m, n, p \in \mathbb{Z}$ and $\theta^{\prime}=\alpha_{n}(\theta)$, the following diagram commutes:

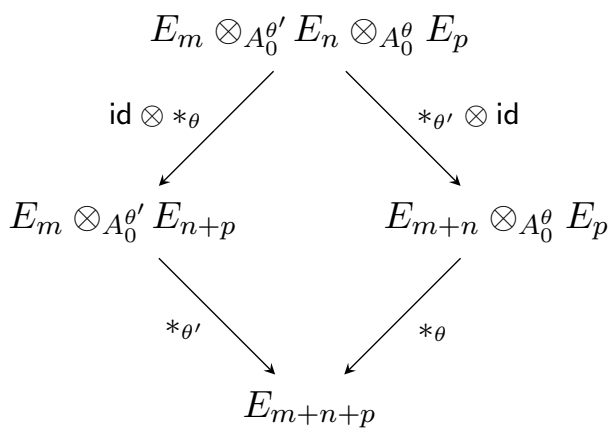

\section{Line bundles on the noncommutative torus}

Let $H_{3}(\mathbb{R})$ be the group of upper triangular matrices

$$
(x, y, t):=\left[\begin{array}{lll}
1 & x & t \\
0 & 1 & y \\
0 & 0 & 1
\end{array}\right], \quad x, y, t \in \mathbb{R} .
$$

With a slight abuse of notations, we identify elements of $\mathcal{U}\left(\mathfrak{h}_{3}(\mathbb{R})\right)$ with left invariant vector fields on $H_{3}(\mathbb{R})$, that are generated by the differential operators

$$
\mathfrak{p}:=\frac{1}{\sqrt{2 \pi}} \frac{\partial}{\partial x}, \quad \mathfrak{q}:=\frac{-i}{\sqrt{2 \pi}}\left(\frac{\partial}{\partial y}+x \frac{\partial}{\partial t}\right), \quad \mathfrak{t}:=\frac{1}{2 \pi i} \frac{\partial}{\partial t} .
$$

The choice of normalization will be clear later on (one can check that (3.1) is satisfied).

Let $H_{3}(\mathbb{Z}):=\left\{(x, y, t) \in H_{3}(\mathbb{R}): x, y, t \in \mathbb{Z}\right\}$. By left invariance, the above vector fields descend to the 3 -dimensional Heisenberg manifold $M_{3}:=H_{3}(\mathbb{Z}) \backslash H_{3}(\mathbb{R})$. Thinking of functions on $K \backslash G$ as left $K$-invariant functions on $G$, we get

$$
C^{\infty}\left(M_{3}\right)=\left\{f \in C^{\infty}\left(\mathbb{R} \times \mathbb{T}^{2}\right): f(x+1, y, t+y)=f(x, y, t)\right\},
$$

where $\mathbb{T}^{2}=\mathbb{R}^{2} / \mathbb{Z}^{2}$ (so $f \in C^{\infty}\left(M_{3}\right)$ is periodic with period 1 in $y$ and $t$ ). The action of central elements $(0,0, t) \in H_{3}(\mathbb{R})$ descends to a principal action of $U(1)$ on $M_{3}$, and $U(1) \backslash M_{3} \simeq \mathbb{T}^{2}$. We identify $C^{\infty}\left(\mathbb{T}^{2}\right)$ with the subset of $f \in C^{\infty}\left(M_{3}\right)$ that do not depend on $t$ (and so are periodic in both $x$ and $y$ ):

$$
C^{\infty}\left(\mathbb{T}^{2}\right)=\left\{f \in C^{\infty}\left(M_{3}\right): f\left(x, y, t+t^{\prime}\right)=f(x, y, t) \forall x, y, t, t^{\prime}\right\} .
$$


Let $A:=C^{\infty}\left(M_{3}\right)$. In the notation of previous section, $A_{0}=C^{\infty}\left(\mathbb{T}^{2}\right)$ and, for $n \neq 0$, every element $f \in A_{n}$ can be written in the form

$$
f(x, y, t)=\sum_{k \in \mathbb{Z}} \tilde{f}\left(x+\frac{k}{n} ; k\right) e^{2 \pi i(k y+n t)}
$$

for a unique Schwartz function $\tilde{f}: \mathbb{R} \times \mathbb{Z} / n \mathbb{Z} \rightarrow \mathbb{C}$. The bijection $A_{n} \rightarrow \mathcal{S}(\mathbb{R} \times \mathbb{Z} / n \mathbb{Z}), f \mapsto \tilde{f}$, is known as Weil-Brezin-Zak transform [12, Section 1.10]. Functions (4.1) can be interpreted as smooth sections of a non-trivial smooth line bundle on $\mathbb{T}^{2}[8]$. The algebra $A_{\bullet}=\bigoplus_{n \in \mathbb{Z}} A_{n}$ is dense in $A$ (in the uniform topology); indeed, by periodicity in $t$, the only weight spaces of $\mathfrak{t}$ appearing in the decomposition of $A$ are those with integer weight. It is strongly $\mathbb{Z}$-graded, which is the algebraic counterpart of the principality of the bundle $M_{3} \rightarrow \mathbb{T}^{2}$ (see, e.g., [1] or [7]).

By point (1) of Corollary 3.3, $A_{0}^{\theta}$ is an associative subalgebra of $A^{\theta}$. By standard Fourier analysis, it is not difficult to verify that $A_{0}^{\theta}$ is generated by two unitary elements, the functions

$$
V(x, y, t):=e^{2 \pi i x}, \quad U(x, y, t):=e^{2 \pi i y},
$$

with relation

$$
U *_{\theta} V=e^{2 \pi i \theta} V *_{\theta} U .
$$

It is then the formal analogue of the smooth algebra of the noncommutative torus.

We can extend $\mathbb{C}[[\nu]]$-linearly the map $f \mapsto \widetilde{f}$ in (4.1) to a bijective map

$$
E_{n}:=A_{n}[[\nu]] \rightarrow \widetilde{E}_{n}:=\mathcal{S}(\mathbb{R} \times \mathbb{Z} / n \mathbb{Z})[[\nu]] .
$$

For $\theta, \theta^{\prime} \in \Theta$, we can define a left action of $A_{0}^{\theta^{\prime}}$ on $\widetilde{E}_{n}$ and a right action of $A_{0}^{\theta}$ on $\widetilde{E}_{n}$ by

$$
a \cdot \tilde{f}:=\widetilde{a *_{\theta^{\prime}} f}, \quad \widetilde{f} \cdot a:=\widetilde{f *_{\theta} a},
$$

for all $\widetilde{f} \in \widetilde{E}_{n}$ and $a \in A_{0}$. A computation using (4.1) gives the explicit formulas (for $n \neq 0$ ):

$$
\begin{array}{llrl}
(U . \tilde{f})(x ; k) & =\widetilde{f}\left(x-\frac{1}{n} ; k-1\right), & & (\widetilde{f} . U)(x ; k)=\widetilde{f}\left(x-\frac{1}{n}-\theta ; k-1\right), \\
(V . \widetilde{f})(x ; k)=e^{2 \pi i\left(x-\frac{k}{n}\right)} e^{-2 \pi i n \theta^{\prime} x} \widetilde{f}(x ; k), & & (\widetilde{f} \cdot V)(x ; k)=e^{2 \pi i\left(x-\frac{k}{n}\right)} \tilde{f}(x ; k),
\end{array}
$$

where for a smooth function $\psi$, by $\psi\left(y+\theta^{\prime}\right)$ we mean the formal power series

$$
\psi\left(y+\theta^{\prime}\right):=e^{\theta^{\prime} \partial_{y}} \psi(y)=\sum_{k \geq 0} \frac{\theta^{\prime k}}{k !} \partial_{y}^{k} \psi(y) .
$$

(If we replace $\theta^{\prime}$ by a real number, although the above series is convergent only for $\psi$ analytic, the formulas (4.4) are well defined for any Schwartz function $\widetilde{f}$.)

By comparing these formulas with equations (2.1)-(2.5) of [18] we recognize the formal analogue of Connes-Rieffel imprimitivity bimodule $E_{g}(\theta)$, in the special case

$$
g=\left(\begin{array}{ll}
1 & 0 \\
n & 1
\end{array}\right)
$$

Finally, for all $\widetilde{f_{1}} \in \widetilde{E}_{n_{1}}$ and $\widetilde{f}_{2} \in \widetilde{E}_{n_{2}}$, one can compute $\widetilde{f_{1} *_{\theta} f_{2}} \in \widetilde{E}_{n_{1}+n_{2}}$ using (4.1) and find the explicit formula

$$
\widetilde{f_{1} *_{\theta} f_{2}}(x ; k)=\sum_{k_{1}+k_{2}=k} \widetilde{f}_{1}\left(\left(1-n_{2} \theta\right) x+\left(1+n_{1} \theta\right) \frac{k_{1} n_{2}-k_{2} n_{1}}{n_{1}\left(n_{1}+n_{2}\right)} ; k_{1}\right) \widetilde{f}_{2}\left(x-\frac{k_{1} n_{2}-k_{2} n_{1}}{n_{2}\left(n_{1}+n_{2}\right)} ; k_{2}\right),
$$


which is valid for $n_{1}, n_{2}, n_{1}+n_{2} \neq 0$. If, on the other hand, $f_{1} \in E_{n}$ and $f_{2} \in E_{-n}(n \neq 0)$, using the identity

$$
\psi(x)=\sum_{m \in \mathbb{Z}} e^{2 \pi i m x} \int_{0}^{1} e^{-2 \pi i m q} \psi(q) \mathrm{d} q,
$$

valid for $\psi \in \mathcal{S}(\mathbb{R})$, we get

$$
f_{1} *_{\theta} f_{2}=\sum_{r, s \in \mathbb{Z}} U^{r} V^{s}\left(\widetilde{f}_{1} \mid \widetilde{f}_{2} \cdot V^{-s} U^{-r}\right)
$$

where

$$
\left(\widetilde{f}_{1} \mid \widetilde{f}_{2}\right):=\sum_{k=1}^{n} \int_{-\infty}^{\infty} \widetilde{f}_{1}((1+n \theta) x ; k) \widetilde{f}_{2}(x ;-k) \mathrm{d} x
$$

Equations (4.6) and (4.7) are a formal version of [19, Proposition 1.2(a)].

We can now give an interpretation to Corollary 3.3: point (2) is the analogue of, e.g., equation (2.6) of [18], stating that the algebra of endomorphisms of a finitely generated projective module over a noncommutative torus is another noncommutative torus with a different deformation parameter; point (4) is the associativity of the pairing of bimodules in [19, Proposition $1.2(\mathrm{~b})]$.

\section{Complex structures and theta functions}

In order to include a complex structure in the construction, it is convenient to start from a different realization of the Heisenberg group and of the principal bundle of previous section.

Let $\tau \in \mathbb{C}$ be a complex number with imaginary part $\Im(\tau)>0$ and $\Lambda:=\mathbb{Z}+\tau \mathbb{Z} \subset \mathbb{C}$ a lattice. We now construct a principal $U(1)$ bundle over the elliptic curve $E_{\tau}:=\mathbb{C} / \Lambda$, isomorphic to $\mathbb{T}^{2}$ if one forgets about the complex structure.

We parametrize the Heisenberg group as follows: we set $H_{3}(\mathbb{R}):=\mathbb{C} \times \mathbb{R}$ with multiplication

$$
\left(z_{1}, t_{1}\right) \cdot\left(z_{2}, t_{2}\right)=\left(z_{1}+z_{2}, t_{1}+t_{2}+\Im\left(\bar{z}_{1} z_{2}\right)\right)
$$

where the bar denotes complex conjugation and $\Im$ the imaginary part. Right invariant vector fields are spanned by

$$
\mathfrak{p}=\sqrt{\frac{\Im(\tau)}{\pi}}\left(\frac{\partial}{\partial z}+\frac{i \bar{z}}{2} \frac{\partial}{\partial t}\right), \quad \mathfrak{q}=\sqrt{\frac{\Im(\tau)}{\pi}}\left(\frac{\partial}{\partial \bar{z}}-\frac{i z}{2} \frac{\partial}{\partial t}\right), \quad \mathfrak{t}=\frac{\Im(\tau)}{\pi i} \frac{\partial}{\partial t},
$$

where we identify elements of $\mathcal{U}\left(\mathfrak{h}_{3}(\mathbb{R})\right)$ with their representation as differential operators.

Let $H_{3}(\mathbb{Z}) \subset H_{3}(\mathbb{R})$ be the subgroup generated by the elements $(1,0)$ and $(\tau, 0)$.

Remark 5.1. For a more explicit description, one can verify that $H_{3}(\mathbb{Z})$ coincides with the group $G:=\left\{g_{m, n, k}:=(m+n \tau,(m n+2 k) \Im(\tau)): m, n, k \in \mathbb{Z}\right\}$. Indeed, from (5.1) we get $g_{m, n, k} g_{m^{\prime}, n^{\prime}, k^{\prime}}=g_{m+m^{\prime}, n+n^{\prime}, k+k^{\prime}-n m^{\prime}}$, proving that $G$ is a group. Clearly $H_{3}(\mathbb{Z}) \subset G$, since $G$ contains the generators $(1,0)$ and $(\tau, 0)$ of $H_{3}(\mathbb{Z})$. On the other hand, $g_{m, n, 0}=(1,0)^{m}(\tau, 0)^{n}$ and $g_{0,0,1}=(1,0) \cdot(\tau, 0) \cdot(-1,0) \cdot(-\tau, 0)$ belong to $H_{3}(\mathbb{Z})$, and since $g_{m, n, k}=g_{m, n, 0}\left(g_{0,0,1}\right)^{k}$, this proves that $H_{3}(\mathbb{Z}) \supset G$. Note that $H_{3}(\mathbb{Z})$ is a proper subgroup of $\Lambda \times \Im(\tau) \mathbb{Z}$, since for example it doesn't contain the element $(0, \Im(\tau))$, and contains properly the group $2(\Lambda \times \Im(\tau) \mathbb{Z})$. 
We define $M_{3}:=H_{3}(\mathbb{R}) / H_{3}(\mathbb{Z})$. As in previous section, the action of central elements $(0, t) \in H_{3}(\mathbb{R})$ descends to a principal action of $U(1)$ on $M_{3}$, and $M_{3} / U(1) \simeq E_{\tau}$. In the notations of Section 2.3, we set $A:=C^{\infty}\left(M_{3}\right)$ and $A_{n}$ as in (3.3), so that $A_{0} \simeq C^{\infty}\left(E_{\tau}\right)$. The right invariance of $f \in A$ under the action of $(0,2 \Im(\tau)) \in H_{3}(\mathbb{Z})$ proves that $f$ is periodic in $t$ with period $2 \Im(\tau)$, hence the differential operator $\mathfrak{t}$ in (5.2) has integer spectrum (this explains the choice of normalization) and the subalgebra $A_{\bullet}=\bigoplus_{n \in \mathbb{Z}} A_{n}$ is dense in $A$.

The map (4.1) is replaced by the bijection (5.3) below.

Proposition 5.2. For all $n \neq 0$ there is a bijection $A_{n} \ni f \mapsto \widetilde{f} \in \mathcal{S}(\mathbb{R} \times \mathbb{Z} / n \mathbb{Z})$ given by

$$
f(z, t)=e^{\pi i n\left\{\frac{1}{\Im(\tau)} t+x y\right\}} \sum_{k \in \mathbb{Z}} e^{2 \pi i k x} \tilde{f}\left(y+\frac{k}{n} ; k\right),
$$

where the real coordinates $(x, y) \in \mathbb{R}^{2}$ are defined by $z:=x+\tau y$.

Proof. This is essentially (4.1) modulo a reparametrization. $f \in A$ iff it is right invariant under the action of the two generators $(1,0)$ and $(\tau, 0)$ of $H_{3}(\mathbb{Z})$. In real coordinates, we get the conditions $f(x+1, y, t-y \Im(\tau))=f(x, y, t)$ and $f(x, y+1, t+x \Im(\tau))=f(x, y, t)$. As mentioned above, $f$ is also $2 \Im(\tau)$-periodic in $t$, and the condition $\mathfrak{t} f=n f$ says that every $f \in A_{n}$ is given by $e^{\pi i n \frac{1}{\Im(\tau)} t}$ times a function of $x, y$. Define $F(x, y)$ implicitly by $f(x, y, z)=$ $e^{\pi i n\left\{\frac{1}{\Im(\tau)} t+x y\right\}} F(x, y)$. The two invariance conditions above become $F(x+1, y)=F(x, y)$ and $F(x, y+1)=F(x, y) e^{-2 \pi i n x}$. From the former, $F(x, y)=\sum_{k \in \mathbb{Z}} e^{2 \pi i k x} F_{k}(y)$ for some functions $F_{k}$. The latter condition gives $F_{k}(y+1)=F_{k+n}(y)$; if we define $\widetilde{f}(y ; k):=F_{k}\left(y-\frac{k}{n}\right)$, the condition becomes $\widetilde{f}(y ; k+n)=\widetilde{f}(y ; k)$. Thus, $\widetilde{f}(y ; k)$ is periodic in $k$ with period $n$. Finally, from [8, Lemma 3.2] it follows that $f$ is $C^{\infty}$ iff $\widetilde{f}$ is Schwartz.

We can apply the same recipe of previous section, and deform the algebra $A$ with the twist $F_{\theta}=\exp \{\theta \mathfrak{p} \otimes \mathfrak{q}\}, \theta \in \Theta$. The advantage is that now $A^{\theta}$ has, besides $A_{0}^{\theta}$, two additional associative subalgebras. The coassociator $\Phi_{\theta, \theta}=e^{\theta^{2} \mathfrak{p} \otimes \mathfrak{t} \otimes \mathfrak{q}}$, cf. (3.7), is 1 on the kernels of $\mathfrak{p}, \mathfrak{t}$ and $\mathfrak{q}$. The second kernel is $A_{0}^{\theta}$, the first and third are related by a conjugation $z \mapsto \bar{z}$. We will focus on the latter.

As in previous section, $A_{0}^{\theta}$ is generated by two unitary functions

$$
U(x, y, t):=e^{2 \pi i x}, \quad V(x, y, t):=e^{2 \pi i y} .
$$

Since $U *_{\theta} V=e^{-\frac{\pi \theta \bar{\tau}}{\Im(\tau)}} U V$ and $V *_{\theta} U=e^{-\frac{\pi \theta \tau}{\Im(\tau)}} V U$, we get the usual noncommutative torus commutation relation $^{1}$

$$
U *_{\theta} V=e^{2 \pi i \theta} V *_{\theta} U .
$$

Let $A_{\text {hol }}:=\operatorname{ker} \mathfrak{q}$ and $A_{\text {hol }}^{\theta}:=\left(A_{\text {hol }}[[\nu]], *_{\theta}\right)$. Since $F_{\theta}$ is 1 on $A_{\text {hol }} \otimes A_{\text {hol }}$, the product is undeformed: $a *_{\theta} b=a b \forall a, b \in A_{\text {hol }}^{\theta}$ and $A_{\text {hol }}^{\theta}$ is a commutative associative subalgebra of $A^{\theta}$.

With an explicit computation we now check that elements of $A_{\text {hol }}$ are (essentially) classical theta functions on the torus. Clearly $A_{0} \cap A_{\text {hol }}=\mathbb{C}$ is the set of constant functions. For $n \neq 0$, the set $A_{n} \cap A_{\text {hol }}$ is described by the following lemma.

Lemma 5.3. If $n<0, A_{\mathrm{hol}} \cap A_{n}=\{0\}$. If $n>0$, elements $f \in A_{\text {hol }} \cap A_{n}$ are in bijection with elements $c_{f} \in \mathbb{C}^{\mathbb{Z} / n \mathbb{Z}}$ via the formula

$$
f(z, t)=e^{\pi i n\left\{\frac{1}{\Im(\tau)} t+x y+\tau y^{2}\right\}} \sum_{k \in \mathbb{Z}} c_{f}(k) q^{k^{2} / n} e^{2 \pi i k z},
$$

where $q:=e^{\pi i \tau}$.

\footnotetext{
${ }^{1}$ The exchange $U \leftrightarrow V$ in (4.2) and (5.4) is needed to get the same commutation relation (4.3) and (5.5).
} 
Proof. With some algebraic manipulation we can rewrite (5.3) as follows

$$
f(z, t)=e^{\pi i n\left\{\frac{1}{\Im(\tau)} t+x y+\tau y^{2}\right\}} \sum_{k \in \mathbb{Z}} q^{k^{2} / n} e^{2 \pi i k z}\left(e^{-\pi i n \tau\left(y+\frac{k}{n}\right)^{2}} \widetilde{f}\left(y+\frac{k}{n} ; k\right)\right) .
$$

In real coordinates, $\mathfrak{q}$ is proportional to the differential operator $\nabla:=\tau \frac{\partial}{\partial x}-\frac{\partial}{\partial y}+\Im(\tau) z \frac{\partial}{\partial t}$. Both the factor $e^{\pi i n\left\{\frac{1}{\Im(\tau)} t+x y+\tau y^{2}\right\}}$ and the holomorphic exponential $e^{2 \pi i k z}$ are in the kernel of such an operator, and since the product in parenthesis in (5.7) only depends on $y$ :

$$
\nabla f(z, t)=-e^{\pi i n\left\{\frac{1}{\Im(\tau)} t+x y+\tau y^{2}\right\}} \sum_{k \in \mathbb{Z}} q^{k^{2} / n} e^{2 \pi i k z} \frac{\partial}{\partial y}\left(e^{-\pi i n \tau\left(y+\frac{k}{n}\right)^{2}} \widetilde{f}\left(y+\frac{k}{n} ; k\right)\right) .
$$

One has $\nabla f=0$ iff $e^{-\pi i n \tau\left(y+\frac{k}{n}\right)^{2}} \widetilde{f}\left(y+\frac{k}{n} ; k\right)=: c_{f}(k)$ does not depend on $y+\frac{k}{n}$, and in this case (5.7) reduces to (5.6). Since $\Im(\tau)>0$, for $n>0$ the function $\widetilde{f}(y ; k)=c_{f}(k) e^{\pi i n \tau y^{2}}$ is of

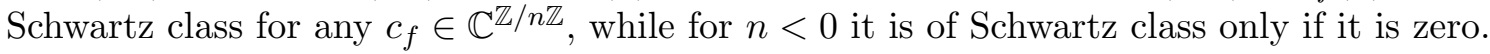

The series in (5.6) are the usual theta functions on $E_{\tau}$. If $n=1$, for example, the series in (5.6) is proportional to the Jacobi's theta function $\vartheta(z ; q)=\sum_{k \in \mathbb{Z}} q^{k^{2} / n} e^{2 \pi i k z}$.

The algebraic structure of theta functions is encoded in the formula (5.8) below.

Proposition 5.4. Let $n_{1}, n_{2}>0$. For all $f_{i} \in A_{\text {hol }} \cap A_{n_{i}}, i=1,2$,

$$
c_{f_{1} f_{2}}(k)=\sum_{\substack{k_{1}, k_{2} \in \mathbb{Z} \\ k_{1}+k_{2}=k}} c_{f_{1}}\left(k_{1}\right) c_{f_{2}}\left(k_{2}\right) q^{\frac{1}{n_{1} n_{2}\left(n_{1}+n_{2}\right)}\left(k_{1} n_{2}-k_{2} n_{1}\right)^{2}},
$$

where $f \mapsto c_{f}$ is the map in (5.6).

Proof. A simple computation using (5.6) and the algebraic identity:

$$
\frac{k_{1}^{2}}{n_{1}}+\frac{k_{2}^{2}}{n_{2}}=\frac{\left(k_{1}+k_{2}\right)^{2}}{n_{1}+n_{2}}+\frac{\left(k_{1} n_{2}-k_{2} n_{1}\right)^{2}}{n_{1} n_{2}\left(n_{1}+n_{2}\right)} .
$$

Remark 5.5. In the $C^{*}$-algebraic setting, for any fixed modular parameter $\tau$, any $g$ as in (1.1) and $\theta \in \mathbb{R}$ solution of $g \theta=\theta$, a ring of "quantum theta functions" $B_{g}(\theta, \tau)$ can be defined as a suitable "holomorphic" subalgebra of the tensor algebra $\bigoplus_{k \geq 0} E_{g}(\theta)^{\otimes k}$ (with tensor product over the algebra $A_{\theta}$ of the noncommutative torus and $E_{g}(\theta)^{\otimes 0}:=A_{\theta}$ ). A product formula for quantum theta functions appeared first in [9] in terms of generators and relations (see also [19]), while an alternative formula which is closer to our notations is [22, equation (7.4)].

For $g$ as in (4.5) and $n=1$, as one can easily check, the product $\left(c_{f_{1}}, c_{f_{2}}\right) \mapsto c_{f_{1} f_{2}}$ defined by (5.8) coincides with [22, equation (7.4)] and $B_{g}(\theta, \tau) \simeq A_{\text {hol }}$ (while $B_{g}(\theta, \tau)$ is a subalgebra of $A_{\text {hol }}$ if $n>1$ in (4.5)). However, this is not surprising since, for $g$ as in (4.5), the only solution to $g \theta=\theta$ is $\theta=0$. The construction in this section, on the other hand, works in a formal setting and there is no constrain on $\theta$. For any $\theta \in \Theta, A^{\theta}$ is well-defined, although not associative, and has an associative (and commutative) subalgebra given by classical theta functions.

\section{On higher rank vector bundles}

In this section, we describe how to derive (formal) imprimitivity bimodules associated to an arbitrary element of $\mathrm{SL}(2, \mathbb{Z})$. 
Let us fix a $g$ as in (1.1), with $d \neq 0$. We denote by $\mathcal{M}_{c, d} \subset C^{\infty}(\mathbb{R} \times \mathbb{T})$ the set of functions satisfying

$$
f(x+d, y)=e^{-2 \pi i c y} f(x, y)
$$

This is a $C^{\infty}\left(\mathbb{T}^{2}\right)$-right module with product given by pointwise multiplication. One can verify that $\mathcal{M}_{c, d}$ is isomorphic to the module of (smooth) sections of a rank $|d|$ vector bundle on $\mathbb{T}^{2}$ as follows. Note that $\mathcal{M}_{c, d} \simeq \mathcal{M}_{-c,-d}$, so from now on we can assume that $d \geq 1$.

That $\mathcal{M}_{0, d}$ is a free module of rank $d$ comes from the following observation.

Remark 6.1. By standard Fourier analysis, every smooth function $\varphi$ with period $d$ can be written (in a unique way) as

$$
\varphi(x)=\sum_{k=1}^{d} e^{2 \pi i \frac{k}{d} x} \varphi_{k}(x)
$$

with $\varphi_{k}$ of period 1 . This gives a $C^{\infty}(\mathbb{R} / \mathbb{Z})$-module isomorphism:

$$
C^{\infty}(\mathbb{R} / d \mathbb{Z}) \rightarrow C^{\infty}(\mathbb{R} / \mathbb{Z}) \otimes \mathbb{C}^{d}, \quad \varphi \mapsto\left(\varphi_{1}, \ldots, \varphi_{d}\right) .
$$

(Functions of period $d$ form a free module of rank $d$ over functions of period 1.)

For the reason above, $\mathcal{M}_{0, d} \simeq \mathcal{M}_{0,1} \otimes \mathbb{C}^{d}$ as modules over $\mathcal{M}_{0,1}=C^{\infty}\left(\mathbb{T}^{2}\right)$.

From now on we forget about free modules and assume that $c \neq 0$ (and $d \geq 1$ as above). Note that the condition $\operatorname{det}(g)=1$ in (1.1) guarantees that $c$ and $d$ are coprime. Vice versa, by Bézout's lemma such a $g$ exists for every coprime $c, d$ (although it is not unique).

Lemma 6.2. Every $n \in \mathbb{Z}$ can be written, in a unique way, as $n=k c+$ md for some $1 \leq k \leq d$ and $m \in \mathbb{Z}$.

Proof. The map $\mathbb{Z}^{2} \ni(k, m) \rightarrow k c+m d \in \mathbb{Z}$ is surjective, due to the identity $n=(-n b) c+(n a) d$ following from the determinant condition in (1.1); since $(k, m)$ and $(k-d, m+c)$ have the same image, one can always choose $1 \leq k \leq d$; restricted to $[1, \ldots, d] \times \mathbb{Z}$ the map is also injective, since $k c+m d=k^{\prime}+m d^{\prime}$ - i.e., $\left(k-k^{\prime}\right) c+\left(m-m^{\prime}\right) d=0$ - implies that $d$ must divide $k-k^{\prime}$. But $\left|k-k^{\prime}\right| \leq d-1$, so it must be $k-k^{\prime}=0$, which also implies $m-m^{\prime}=0$.

Remark 6.1 can then be rephrase as follows.

Lemma 6.3. Every $\varphi \in C^{\infty}(\mathbb{R} / d \mathbb{Z})$ can be written in a unique way as

$$
\varphi(x)=\sum_{k=1}^{d} e^{2 \pi i \frac{c}{d} k x} \varphi_{k}(x)
$$

with $\varphi_{1}, \ldots, \varphi_{d} \in C^{\infty}(\mathbb{R} / \mathbb{Z})$.

Proof. From Lemma 6.2, we can write the Fourier series of $\varphi$ as

$$
\varphi(x)=\sum_{n \in \mathbb{Z}} e^{2 \pi i \frac{1}{d} n x} \hat{\varphi}_{k}=\sum_{k=1}^{d} e^{2 \pi i \frac{c}{d} k x}\left(\sum_{m \in \mathbb{Z}} e^{2 \pi i m x} \hat{\varphi}_{k}\right)
$$

and called $\varphi_{k}(x)=\sum_{m \in \mathbb{Z}} e^{2 \pi i m x} \hat{\varphi}_{k}$ we get the desired result. 
Proposition 6.4. $\mathcal{M}_{c, d}$ is isomorphic to the $C^{\infty}\left(\mathbb{T}^{2}\right)$-module of smooth sections of the vector bundle

$$
\begin{gathered}
\left(\mathbb{T} \times \mathbb{R} \times \mathbb{C}^{d}\right) / \mathbb{Z} \\
\downarrow \\
\mathbb{T} \times \mathbb{R} / \mathbb{Z}=\mathbb{T}^{2},
\end{gathered}
$$

where the action of $\mathbb{Z}$ on $\mathbb{T} \times \mathbb{R} \times \mathbb{C}^{d}$ is generated by the map

$$
\left(x, y ; v_{1}, v_{2}, \ldots, v_{d}\right) \mapsto\left(x, y+1 ; e^{2 \pi i c x} v_{d}, v_{1}, \ldots, v_{d-1}\right) .
$$

Proof. Any $f \in \mathcal{M}_{c, d}-$ since $e^{2 \pi i \frac{c}{d} x y} f(x, y)$ is periodic in $x$ with period $d$ - can be written (in a unique way) in the form $f(x, y)=e^{-2 \pi i \frac{c}{d} x y} \sum_{k=1}^{d} e^{2 \pi i \frac{c}{d} k x} f_{k}(x, y)$, where $f_{1}, \ldots, f_{d}$ have period 1 in $x$. From (6.1) we get the conditions

$$
f_{k}(x, y+1)=f_{k-1}(x, y), \quad \forall k \neq 1, \quad f_{1}(x, y+1)=e^{2 \pi i c x} f_{d}(x, y) .
$$

We may then think of $\left(f_{1}, \ldots, f_{d}\right)$ as a section of the vector bundle (6.2). More precisely, the corresponding section is the map

$$
\mathbb{T}^{2} \ni[x, y] \mapsto\left[[x], y ; f_{1}(x, y), \ldots, f_{d}(x, y)\right] \in\left(\mathbb{T} \times \mathbb{R} \times \mathbb{C}^{d}\right) / \mathbb{Z} .
$$

The transformation $f \mapsto\left(f_{1}, \ldots, f_{d}\right)$ is the desired $C^{\infty}\left(\mathbb{T}^{2}\right)$-module isomorphism.

Let $U, V$ be the generators of $C^{\infty}\left(\mathbb{T}^{2}\right)$ in $(4.2)$, and $U^{\prime}, V^{\prime}$ the $C^{\infty}\left(\mathbb{T}^{2}\right)$-linear endomorphisms of $\mathcal{M}_{c, d}$ given by

$$
\left(U^{\prime} f\right)(x, y):=e^{2 \pi i a y} f(x+b, y), \quad\left(V^{\prime} f\right)(x, y):=e^{2 \pi i \frac{1}{d} x} f(x, y) .
$$

(Here $a, b$ are the elements in the first row of (1.1).) The operators $U^{\prime}, V^{\prime}$ are unitary if we equip $\mathcal{M}_{c, d}$ with the inner product given by $\left\langle f_{1}, f_{2}\right\rangle:=\int_{[0, d] \times[0,1]} f_{1}(x, y)^{*} f_{2}(x, y) \mathrm{d} x \mathrm{~d} y$, and satisfy the defining relation of the rational noncommutative torus with deformation parameter $b / d$, namely $U^{\prime} V^{\prime}=e^{2 \pi i \frac{b}{d}} V^{\prime} U^{\prime}$. Let us denote by $B_{b / d}$ the algebra of power series in $U^{\prime}, V^{\prime}$ with rapid decay coefficients.

In order to apply the deformation machinery of Section 3 we need an action of $\mathfrak{h}_{3}(\mathbb{R})$. The pointwise product gives a map $\mathcal{M}_{c, d} \times \mathcal{M}_{c^{\prime}, d^{\prime}} \rightarrow \mathcal{M}_{c d^{\prime}+c^{\prime} d, d d^{\prime}}$. An action of $\mathfrak{h}_{3}(\mathbb{R})$ by derivations is

$$
\mathfrak{p} f=\frac{1}{\sqrt{2 \pi}} \frac{\partial}{\partial x} f, \quad \mathfrak{q} f=\frac{-i}{\sqrt{2 \pi}}\left(\frac{\partial}{\partial y}+2 \pi i \frac{c}{d} x\right) f, \quad \mathfrak{t} f=\frac{c}{d} f
$$

for all $f \in \mathcal{M}_{c, d}$. The condition that $\mathfrak{q}$ is a derivation (i.e., satisfies the Leibniz rule) fixes the constant in front of the factor $x$, and consequently the normalization of $\mathfrak{t}$. In particular $\mathcal{M}_{0,1}=C^{\infty}\left(\mathbb{T}^{2}\right)$ is in the kernel of $\mathfrak{t}$.

A compatible action on $B_{b / d} \subset \operatorname{End}_{C^{\infty}\left(\mathbb{T}^{2}\right)}\left(\mathcal{M}_{c, d}\right)$ is given by commutators: for all primitive $X \in \mathfrak{h}_{3}(\mathbb{R})$ and all $\xi \in B_{b / d}, X(\xi)$ is the endomorphism defined by $X(\xi) f:=X(\xi f)-\xi X(f)$ for all $f \in \mathcal{M}_{c, d}$. With a simple computation one checks that $\mathfrak{t}$ is mapped to 0 , while $\mathfrak{p} \mapsto \frac{1}{\sqrt{2 \pi}} \delta_{V^{\prime}}$ and $\mathfrak{q} \mapsto \frac{-i}{\sqrt{2 \pi}} \delta_{U^{\prime}}$, where $\delta_{U^{\prime}}, \delta_{V^{\prime}}$ are the derivations defined on generators by

$$
\delta_{U^{\prime}}\left(U^{\prime}\right)=\frac{2 \pi i}{d} U^{\prime}, \quad \delta_{U^{\prime}}\left(V^{\prime}\right)=0, \quad \delta_{V^{\prime}}\left(U^{\prime}\right)=0, \quad \delta_{V^{\prime}}\left(V^{\prime}\right)=\frac{2 \pi i}{d} V^{\prime} .
$$

We can now use the twisting element in Section 3 to construct, for any $\theta, \theta^{\prime} \in \Theta$, two new algebras $A_{0}^{\theta}:=\left(C^{\infty}\left(\mathbb{T}^{2}\right)[[\nu]], *_{\theta}\right)$ and $B_{b / d}^{\theta^{\prime}}:=\left(B_{b / d}[[\nu]], *_{\theta^{\prime}}\right)$ and to deform $E_{c, d}:=\mathcal{M}_{c, d}[[\nu]]$ into a left $B_{b / d^{\prime}}^{\theta^{\prime}}$-module and right $A_{0}^{\theta}$-module. 
Note that $A_{0}^{\theta}$ and $B_{b / d}^{\theta^{\prime}}$ are both associative (they are in the kernel of $\mathfrak{t}$, hence the coassociator is trivial), the former is generated by the two unitaries $U$ and $V$ with relation (4.3), the latter by the two unitaries $U^{\prime}$ and $V^{\prime}$ with relation

$$
U^{\prime} *_{\theta^{\prime}} V^{\prime}=e^{2 \pi i\left(d^{-2} \theta^{\prime}+\frac{b}{d}\right)} V^{\prime} *_{\theta^{\prime}} U^{\prime} .
$$

The action $\alpha$ in (3.2) extends in an obvious way to an action of $\mathbb{Q}$ on $\Theta$, and from Lemma 3.1 we derive the following analogue of Proposition 3.2.

Proposition 6.5. The left $B_{b / d}^{\theta^{\prime}}$ action and right $A_{0}^{\theta}$ action on $E_{c, d}$ commute, i.e.,

$$
\left(\xi *_{\theta^{\prime}} f_{1}\right) *_{\theta} f_{2}=\xi *_{\theta^{\prime}}\left(f_{1} *_{\theta} f_{2}\right) \quad \forall \xi \in B_{b / d}^{\theta^{\prime}}, \quad f_{1} \in E_{c, d}, \quad f_{2} \in A_{0}^{\theta},
$$

if and only if $\theta^{\prime}=\alpha_{\frac{c}{d}}(\theta)$.

Proof. If $\theta^{\prime}=\alpha_{\frac{c}{d}}(\theta)$, since $\mathfrak{t} f=\frac{c}{d} f$ for all $f \in E_{c, d}$, we deduce that $\Phi_{\theta, \theta^{\prime}}$ is the identity on $\xi \otimes f_{1} \otimes f_{2}$ and then (6.4) holds. On the other hand,

$$
m(m \otimes \mathrm{id})\left(\Phi_{\theta, \theta^{\prime}}-1\right)\left(V^{\prime} \otimes f \otimes U\right)=\left(e^{2 \pi i d^{-1}\left(1+\frac{c}{d} \theta\right)\left\{\alpha_{c / d}(\theta)-\theta^{\prime}\right\}}-1\right) V^{\prime} f U
$$

is zero for all $f \in E_{c, d}$ only if $\theta^{\prime}=\alpha_{\frac{c}{d}}(\theta)$.

Note that if $\theta^{\prime}=\alpha_{\frac{c}{d}}(\theta)$, the parameter in (6.3) is

$$
d^{-2} \theta^{\prime}+\frac{b}{d}=\frac{a \theta+b}{c \theta+d}=g \theta
$$

as expected.

The action of the two algebras on $E_{c, d}$ can be explicitly computed on generators. The left action of $U^{\prime}$ and the right action of $V$ are undeformed, while for the remaining two generators one gets

$$
\begin{aligned}
& \left(f *_{\theta} U\right)(x, y)=e^{2 \pi i y} f(x-\theta, y), \\
& \left(V^{\prime} *_{\theta^{\prime}} f\right)(x, y)=e^{2 \pi i \frac{1}{d} x} e^{-2 \pi i c d^{-2} \theta^{\prime} x} f\left(x, y-d^{-1} \theta^{\prime}\right) .
\end{aligned}
$$

It remains to compare what we obtained with the well-known formulas that one has in the $C^{*}$-algebraic setting, cf., e.g., equations (2.1)-(2.5) of [18]. This can be done by means of the transform $E_{c, d} \rightarrow \widetilde{E}_{c, d}:=\mathcal{S}(\mathbb{R} \times \mathbb{Z} / c \mathbb{Z})[[\nu]], f \mapsto \widetilde{f}$, defined by

$$
f(x, y)=\sum_{k \in \mathbb{Z}} \tilde{f}\left(x+k \frac{d}{c} ; k\right) e^{2 \pi i k y}
$$

and similar to (4.1) (except for $\frac{1}{n}$ replaced by $\frac{d}{c}$ ). Under this transform, the left/right module structure become:

$$
\begin{array}{llrl}
\widetilde{U^{\prime} *_{\theta^{\prime}} f}(x ; k) & =\widetilde{f}\left(x-\frac{1}{c} ; k-a\right), & \widetilde{f *_{\theta} U}(x ; k) & =\widetilde{f}\left(x-\frac{d}{c}-\theta ; k-1\right), \\
\widetilde{V^{\prime} *_{\theta^{\prime}} f}(x ; k) & =e^{2 \pi i \frac{1}{d}\left(x-k \frac{d}{c}\right)} e^{-2 \pi i c d^{-2} \theta^{\prime} x} \widetilde{f}(x ; k), & \widetilde{f *_{\theta} V}(x ; k) & =e^{2 \pi i\left(x-k \frac{d}{c}\right)} \widetilde{f}(x ; k),
\end{array}
$$

for all $\widetilde{f} \in \widetilde{E}_{c, d}$. These formulas reduce to equations (2.1)-(2.5) of [18] if $\theta^{\prime}=\alpha_{\frac{c}{d}}(\theta)$ and after replacing formal deformation parameters by irrational numbers. 


\section{References}

[1] Arici F., D'Andrea F., Landi G., Pimsner algebras and noncommutative circle bundles, arXiv:1506.03109.

[2] Arici F., Kaad J., Landi G., Pimsner algebras and Gysin sequences from principal circle actions, J. Noncommut. Geom., to appear, arXiv:1409.5335.

[3] Brzeziński T., Majid S., Quantum group gauge theory on quantum spaces, Comm. Math. Phys. 157 (1993), 591-638, hep-th/9208007.

[4] Chari V., Pressley A., A guide to quantum groups, Cambridge University Press, Cambridge, 1994.

[5] Connes A., $C^{*}$ algèbres et géométrie différentielle, C. R. Acad. Sci. Paris Sér. A-B 290 (1980), A599-A604, hep-th/0101093.

[6] Connes A., Noncommutative geometry, Academic Press, Inc., San Diego, CA, 1994.

[7] D'Andrea F., Topics in noncommutative geometry, Lecture Notes for the Autumn School "From Poisson Geometry to Quantum Fields on Noncommutative Spaces" (Würzburg, 2015), arXiv:1510.07271.

[8] D'Andrea F., Fiore G., Franco D., Modules over the noncommutative torus and elliptic curves, Lett. Math. Phys. 104 (2014), 1425-1443, arXiv:1307.6802.

[9] Dieng M., Schwarz A., Differential and complex geometry of two-dimensional noncommutative tori, Lett. Math. Phys. 61 (2002), 263-270, math.QA/0203160.

[10] Drinfeld V.G., Quasi-Hopf algebras, Leningrad Math. J. 1 (1989), 1419-1457.

[11] Drinfeld V.G., On quasitriangular quasi-Hopf algebras and on a group that is closely connected with $\operatorname{Gal}(\overline{\mathbf{Q}} / \mathbf{Q})$, Leningrad Math. J. 2 (1990), 829-860.

[12] Folland G.B., Harmonic analysis in phase space, Annals of Mathematics Studies, Vol. 122, Princeton University Press, Princeton, NJ, 1989.

[13] Gracia-Bondía J.M., Várilly J.C., Figueroa H., Elements of noncommutative geometry, Birkhäuser Advanced Texts: Basler Lehrbücher, Birkhäuser Boston, Inc., Boston, MA, 2001.

[14] Hajac P.M., Strong connections on quantum principal bundles, Comm. Math. Phys. 182 (1996), 579-617, hep-th/9406129.

[15] Landi G., An introduction to noncommutative spaces and their geometries, Lecture Notes in Physics Monographs, Vol. 51, Springer-Verlag, Berlin, 1997, hep-th/9701078.

[16] Mac Lane S., Categories for the working mathematician, Graduate Texts in Mathematics, Vol. 5, 2nd ed., Springer-Verlag, New York, 1998.

[17] Majid S., Foundations of quantum group theory, Cambridge University Press, Cambridge, 1995.

[18] Plazas J., Arithmetic structures on noncommutative tori with real multiplication, Int. Math. Res. Not. (2008), Art. ID rnm147, 41 pages, math.QA/0610127.

[19] Polishchuk A., Schwarz A., Categories of holomorphic vector bundles on noncommutative two-tori, Comm. Math. Phys. 236 (2003), 135-159, math.QA/0211262.

[20] Rieffel M.A., $C^{*}$-algebras associated with irrational rotations, Pacific J. Math. 93 (1981), 415-429.

[21] Rieffel M.A., The cancellation theorem for projective modules over irrational rotation $C^{*}$-algebras, Proc. London Math. Soc. 47 (1983), 285-302.

[22] Vlasenko M., The graded ring of quantum theta functions for noncommutative torus with real multiplication, Int. Math. Res. Not. 2006 (2006), Art. ID 15825, 19 pages, math.QA/0601405. 